\title{
Peeling linear inversion of upper mantle velocity structure with receiver functions*
}

\author{
Xuzhang Shen ${ }^{1, *}$ and Huilan Zhou ${ }^{2}$ \\ ${ }^{1}$ Lanzhou Institute of Seismology, China Earthquake Administration, Lanzhou 730000, China \\ ${ }^{2}$ Laboratory of Computational Geodynamics, Graduate University of Chinese Academy of \\ Sciences, Beijing 100049, China
}

\begin{abstract}
A peeling linear inversion method is presented to study the upper mantle (from Moho to $800 \mathrm{~km}$ depth) velocity structures with receiver functions. The influences of the crustal and upper mantle velocity ratio error on the inversion results are analyzed, and three valid measures are taken for its reduction. This method is tested with the IASP91 and the PREM models, and the upper mantle structures beneath the stations GTA, LZH, and AXX in northwestern China are then inverted. The results indicate that this inversion method is feasible to quantify upper mantle discontinuities, besides the discontinuities between $3 h_{\mathrm{M}}\left(h_{\mathrm{M}}\right.$ denotes the depth of Moho) and $5 h_{\mathrm{M}}$ due to the interference of multiples from Moho. Smoothing is used to overcome possible false discontinuities from the multiples and ensure the stability of the inversion results, but the detailed information on the depth range between $3 h_{\mathrm{M}}$ and $5 h_{\mathrm{M}}$ is sacrificed.
\end{abstract}

Key words: upper mantle; receiver function; peeling; linear inversion

CLC number: P315.3 Document code: A

\section{Introduction}

Inversion of the upper mantle velocity structure using observed receiver function $(\mathrm{ORF}) r^{\mathrm{o}}(t)$ is not easy because the amplitude of the first-transmission seismic phases and the crustal multiples are much larger than those of $\mathrm{P}_{410} \mathrm{~s}$ and $\mathrm{P}_{660} \mathrm{~s}$, and conception about the upper mantle in $r^{\mathrm{o}}(t)$ is not clear. Therefore, the inversion depth of many previous results only reached the Moho and the uppermost mantle. Some studies, however, attempted to extract more information from the deeper upper mantle. Liu et al. (1997) used a complex spectrum ratio of the receiver functions to invert the crust and uppermost mantle structures below 10 China Digital Seismograph Network stations down to a depth of $100 \mathrm{~km}$. Vinnik et al. (2004) inverted the velocity structure down to $150 \mathrm{~km}$ depth beneath central Tianshan using $\mathrm{P}$ and $\mathrm{S}$ receiver functions. Julià et al.

\footnotetext{
* Received 1 October 2011; accepted in revised form 25 December 2011; published 10 February 2012.

* Corresponding author. e-mail: shenxzh@gmail.com

(c) The Seismological Society of China, Institute of Geophysics, China Earthquake Administration, and Springer-Verlag Berlin Heidelberg 2012
}

(2005) combined the receiver functions with surface wave to invert the crustal and upper mantle velocity structure of eastern Africa down to the depth of 300 $\mathrm{km}$. Zhou and Yang (2003) proposed a peeling genetic algorithm of $r^{\circ}(t)$ and extended the inversion depth to $700 \mathrm{~km}$. This method divides the crust and upper mantle into several parts and inverts their velocities step by step from the crust to the depth of $700 \mathrm{~km}$, which presents a new way to study the deep discontinuities in the upper mantle. But the efficiency of this method is not satisfactory due to the excessive parameters used in the inversion. In addition, the results of deeper structures are distorted for the cases with incorrect or unstable shallow structures. Although the genetic algorithm method is very good for searching for the global optimal solution, it inevitably falls into the local optimal solution.

Here, the peeling linear inversion method of $r^{\mathrm{o}}(t)$ tries to investigate the upper mantle velocity structure by fixing the crustal structure from previous results. The velocity between the Moho and $800 \mathrm{~km}$ depth is inverted by peeling part of $r^{\mathrm{o}}(t)$ corresponding to the crust. A simple initial model is used in the inversion. 
Based on numerical tests, the influence of the error of the crustal and upper mantle velocity ratio is analyzed, and it is then reduced by smoothing some special depth ranges.

The method is tested with two global Earth models. The ORFs in the stations GTA, LZH, and AXX in northwestern China are used to invert the upper mantle structure beneath the stations.

\section{Method}

\subsection{Concept of peeling}

The time windows of $r^{\circ}(t)$ should be set from initial $\mathrm{P}$ arrival to $80 \mathrm{~s}$ to study the crustal and upper mantle velocity structure. The phase amplitude of $r^{\mathrm{o}}(t)$ in the upper mantle discontinuities is small even if the $\mathrm{P}_{660} \mathrm{~s}$ amplitude, the strongest phase of the upper mantle discontinuities, is only one-third of that of the $\mathrm{P}_{\mathrm{MS}}$. Information on the upper mantle discontinuities in ORFs is very minimal due to the existence of noise, thus, identifying this weak signal is oftentimes difficult. The fitting window will concentrate in the 0-20 s range during inversion when the crustal and the upper mantle structures are completely inverted. This time window just contains the information about the crust and uppermost mantle, so the upper mantle structure cannot be inverted effectively. The peeling method is a possible solution for this problem. Based on previous crustal structure results, the upper mantle structure is inverted using linear inversion. The steps are as follows:

1) The crustal structure obtained from the receiver functions and surface wave dispersion curves in many regions can be used to construct initial model of the crust. During the inversion, the crustal structure is fixed by adjusting the parameters.

2) The upper mantle structure is generally identical for different regions compared with crustal structure, so a common and simple initial model of the upper mantle is used. $r_{\text {crust }}(t)$, the part of $r^{\circ}(t)$ corresponding to the crust, is nearly invariable because of fixed crust model, so the upper mantle structure is adjusted to fit $r_{\text {mantle }}(t)=r^{\mathrm{o}}(t)-r_{\text {crust }}(t)$, the residual part of $r^{\mathrm{o}}(t)$.

2.2 Linear inversion of the upper mantle velocity structure

\subsubsection{Program and parameters}

The program package PROGRAMS.330 (Herrmann and Ammon, 2002) is adopted to invert the upper mantle structures with $r^{\mathrm{o}}(t)$. The thickness or the velocity of each layer $m_{i}$ in the inversion can be changed, and the parameters are adjusted so as to control the variation of the unknown thickness or velocity at a certain depth. The detailed information about the parameters is presented as following.

The relationship among the vectors of the velocity model $\boldsymbol{M}\left(m_{1}, m_{2}, \cdots, m_{i}\right)$, initial model $\boldsymbol{M}_{0}$, and residual $S$ between $r^{\circ}(t)$ and the synthetic receiver function predicted from the theoretical model can be expressed as

$$
\left(\begin{array}{c}
\boldsymbol{D} \\
\sigma \boldsymbol{\Delta}
\end{array}\right) \boldsymbol{M}=\left(\begin{array}{c}
S \\
0
\end{array}\right)+\left(\begin{array}{c}
\boldsymbol{D} \boldsymbol{M}_{0} \\
0
\end{array}\right)
$$

where $\boldsymbol{D}$ is a matrix containing the partial derivatives of the waveform with respect to changes in the layer velocities for model $\boldsymbol{M}_{0}, \boldsymbol{\Delta}$ is the roughness matrix of the model, and $\sigma$ is a balancing factor between the variance of the model and fitness of the observed data. $\boldsymbol{\Delta}=\boldsymbol{W}_{1} \boldsymbol{W}_{2}$, where $\boldsymbol{W}_{1}$ is a general smoothing matrix, which minimizes the difference between adjacent values of the change in $m_{i}$ from the initial model, and is expressed as

$$
\boldsymbol{W}_{1}=\left(\begin{array}{ccccc}
1 & -1 & 0 & \cdots & 0 \\
0 & 1 & -1 & \cdots & 0 \\
\vdots & \vdots & \vdots & \vdots & \vdots \\
0 & 0 & \cdots & 1 & -1 \\
0 & 0 & 0 & \cdots & 1
\end{array}\right)
$$

In this form, $\boldsymbol{W}_{1}$ can force a degree of smoothness on the changes in $m_{i}$. If $\boldsymbol{W}_{1}=\boldsymbol{I}$, there is no smoothness constraint in the inversion. $\boldsymbol{W}_{2}=\operatorname{diag}\left\{\chi_{1}^{-1}, \chi_{2}^{-1}, \chi_{3}^{-1}\right.$, $\left.\cdots, \chi_{n}^{-1}\right\}$ is constructed by weighted factor $\chi_{i}$ for each layer, and used to provide a relative weight to the constraints. For example, to force a sharp discontinuity at a given boundary when smoothing constraint is employed, $\chi_{i}^{-1}$ is made small. To select appropriate values of $\boldsymbol{W}_{1}$ and $\boldsymbol{W}_{2}$, the value of $m_{i}$ can be changed sharply at some depths where discontinuity might exist. The model can also be smoothed at some depths where no discontinuity might be presented.

\subsubsection{Initial model of the upper mantle}

The linear inversion method result depends greatly on the initial model, so constructing an initial model is very critical. Two initial models are constructed for the inversion in our study.

1) The first initial model (Figure 1a). Many studies have shown that " 410 " and "660" are global discontinuities, and other discontinuities in the upper mantle have been detected (Anderson and Bass, 1986; Shearer, 1990; Shearer and Flanagan, 1996) although they are 
not global. The initial model (from Moho to $800 \mathrm{~km}$ ) is obtained from IASP91 model (Kennett and Engdahl, 1991) without first-order discontinuity. The velocity values around the depths of 410 and $660 \mathrm{~km}$ are permitted to change sharply in order to search for the discontinuities. Details on the initial steps are as follows: firstly, the entire upper mantle is divided into many thin layers from Moho to $800 \mathrm{~km}$ depth. The thickness of each layer $h_{i}$ is determined according to the resolution of $r^{\circ}(t)$
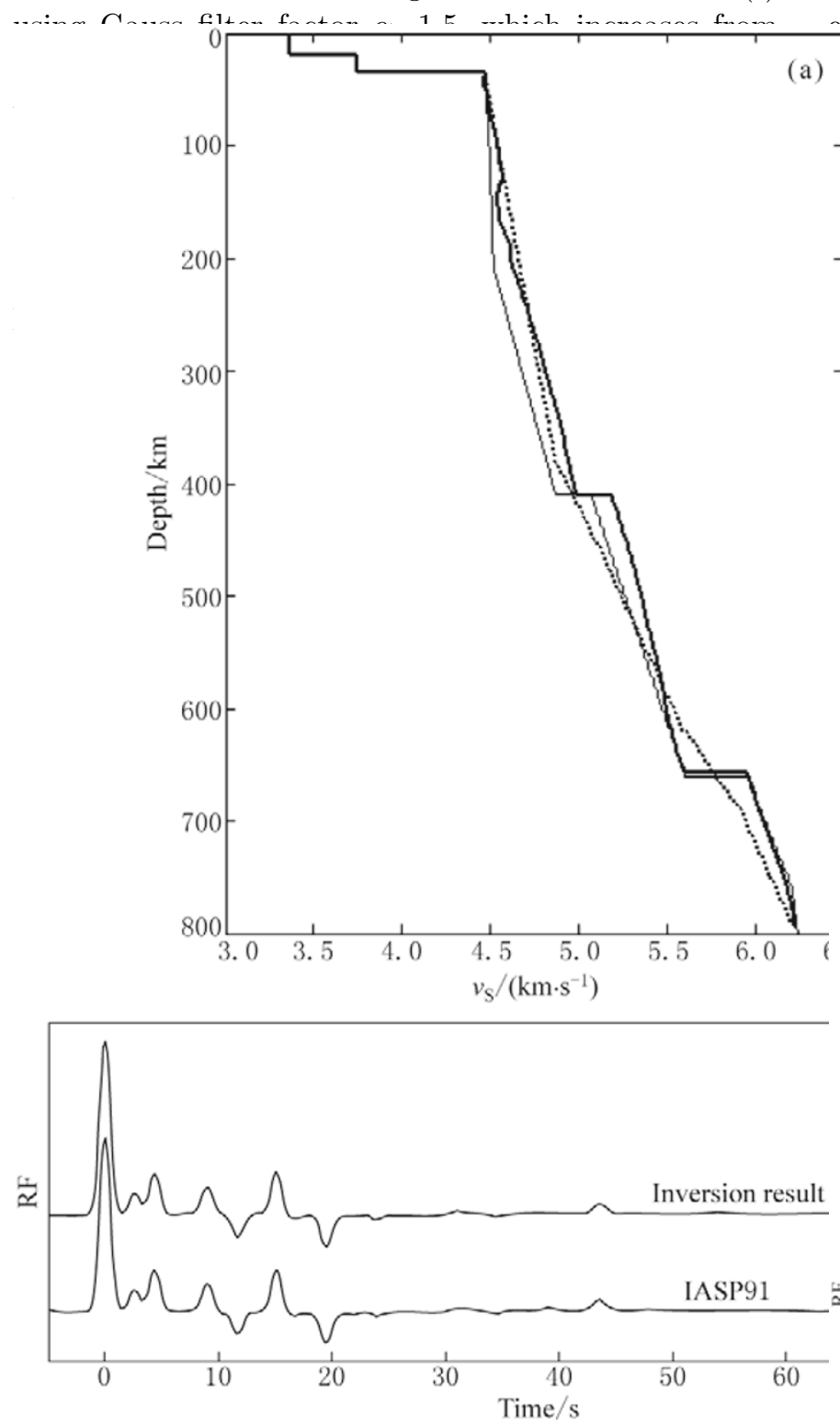

Figure 1 (a) Numerical test of the linear inversion method for the IASP91 model. Thin line stands for IASP91 model, dotted line for initial model, and solid thick line for inversion model. (b) Receiver functions of the inversion and IASP91 models. and $620-700 \mathrm{~km}, v_{\mathrm{S}}$ can be changed sharply by introducing a large $\chi_{i}$. If the stacking result of the ORFs shows other discontinuities at some depths, the velocities can be permitted to change largely around these depths by controlling the $\chi_{i}$ value. The model is flattened to calculate the synthetic receiver function in the inversion because the IASP91 model is spherical. The final inversion result is still a spherical model.

2) As shown in Figure 2a, the second initial mod1 … previous result from the upper mantle re study area. In the present paper, the reen Moho and $350 \mathrm{~km}$ depth is used to nitial model based on the inversion results wave dispersion curve combined with the al structure.
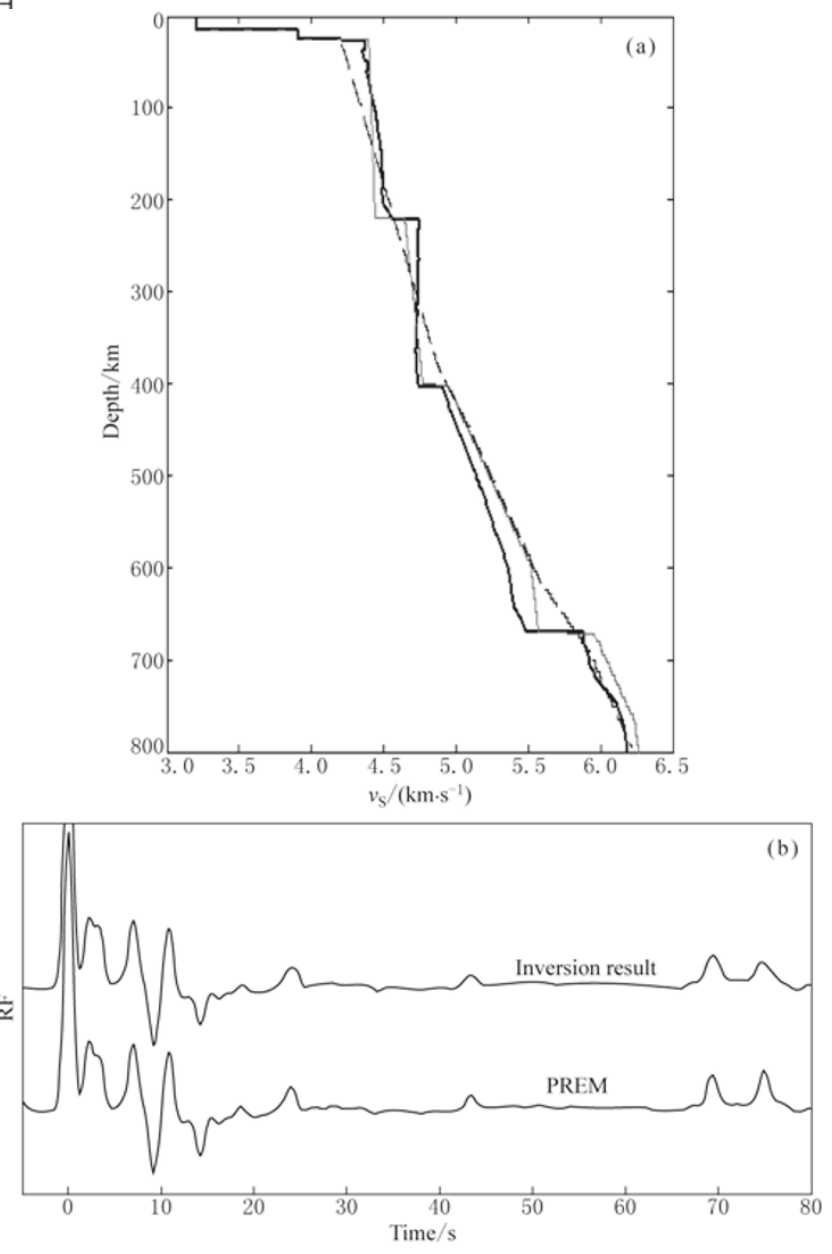

Figure 2 (a) Numerical test of the linear inversion method for the PREM. Gray line stands for PREM, dashed line for initial model, and black line for inversion model. (b) Receiver functions of the inversion and PREM models. 


\subsubsection{Detailed steps}

The linear inversion is carried out according the following two steps. Firstly, the approximate depth range in the upper mantle is estimated in reference to the stacking results of $r^{\circ}(t)$ after the moveout correction (Yuan et al., 1997). $\chi_{i}$ is set to 5.0 in this depth range, and is fixed to 1.0 in the other depth ranges in the upper mantle, whereas $\chi_{i}$ is taken as 0 in the crust, which ensures that the velocity values are not changed during the inversion process. A total of 40 iterations are made for the initial model in linear inversion. Then, the crustal velocity is also iterated in the last 10 times with $\chi_{i}=0.2$ in the crust, and $\chi_{i}=1$ in the upper mantle. These values can reduce the effect of deviation from the crustal structure. Secondly the "410" or " 660 " discontinuity in the above inversion result is often a gradient layer instead of a first-order discontinuity. The mean value of the depths of the gradient layer is considered as the depth of the discontinuity. Subsequently, the depths of the discontinuities are input into initial models to construct a new initial model. The upper mantle velocity is inverted based on this initial model. In the former 40 iterations, $v_{\mathrm{S}_{i}}$ and $h_{i}$ are changed, $\chi_{i}$ is fixed to 5 , and smoothing is not applied. In the 41st to 50th iterations, only the $v_{\mathrm{S}_{i}}$ values in the crust and upper mantle are considered. The final inversion results are the crustal and upper mantle velocity model.

\subsection{Error analysis}

\subsubsection{Error in upper mantle velocity}

The covariance matrix of the inversion parameters in equation (1) can be described as follows (Ammon et al., 1990):

$$
\begin{aligned}
{[\operatorname{cov} \boldsymbol{M}] } & =\left(\boldsymbol{\Delta}^{-1} \boldsymbol{V}\left(\boldsymbol{\Lambda}^{2}+\sigma^{2} \boldsymbol{I}\right)^{-1} \boldsymbol{\Lambda} \boldsymbol{U}^{\mathrm{T}}\right) \delta \boldsymbol{d} \delta \boldsymbol{d}^{\mathrm{T}} \\
& {\left[\boldsymbol{\Delta}^{-1} \boldsymbol{V}\left(\boldsymbol{\Lambda}^{2}+\sigma^{2} \boldsymbol{I}\right)^{-1} \boldsymbol{\Lambda} \boldsymbol{U}^{\mathrm{T}}\right]^{\mathrm{T}}, }
\end{aligned}
$$

where $\delta \boldsymbol{d}$ is the error of $\boldsymbol{d}, \boldsymbol{U}$ and $\boldsymbol{V}$ are orthographical matrix arising from singular value decomposition of $\boldsymbol{D} \boldsymbol{\Delta}^{-1} . \boldsymbol{D} \boldsymbol{\Delta}^{-1}=\boldsymbol{U} \boldsymbol{\Lambda} \boldsymbol{V}^{\mathrm{T}} . \boldsymbol{\Lambda}$ is a diagonal matrix composed of the positive eigenvalue of matrix $\left(\boldsymbol{D} \boldsymbol{\Delta}^{-1}\right)\left(\boldsymbol{D} \boldsymbol{\Delta}^{-1}\right)^{\mathrm{T}}$.

Assuming that the observed data are independent of each other but with the same variance $E^{2}$, $\delta \boldsymbol{d} \delta \boldsymbol{d}^{\mathrm{T}}=E^{2} \boldsymbol{I}$ yields

$$
\begin{gathered}
{[\operatorname{cov} \boldsymbol{M}]=E^{2} \boldsymbol{\Delta}^{-1} \boldsymbol{V}\left(\boldsymbol{\Lambda}^{2}+\sigma^{2} \boldsymbol{I}\right)^{-1}} \\
\boldsymbol{\Lambda}^{2}\left(\boldsymbol{\Lambda}^{2}+\sigma^{2} \boldsymbol{I}\right)^{-1} \boldsymbol{V}^{\mathrm{T}}\left(\boldsymbol{\Delta}^{-1}\right)^{\mathrm{T}} .
\end{gathered}
$$

The velocity covariance matrix can be calculated using equation (4) if $E^{2}$ is known in advance. $E^{2}$ can be estimated according to the signal-to-noise ratio (SNR) of the observed waveforms. The waveforms of the $R$ and $Z$ components can be expressed as

$$
\left\{\begin{array}{l}
R(t)=R_{\mathrm{S}}(t)+R_{\mathrm{N}}(t) \\
Z(t)=Z_{\mathrm{S}}(t)+Z_{\mathrm{N}}(t)
\end{array} .\right.
$$

The subscripts ' $\mathrm{N}$ ' and ' $\mathrm{S}$ ' represent the noise and signal, respectively. Then, we have

$$
r^{\mathrm{o}}(t)=r_{\mathrm{S}}^{\mathrm{o}}(t)+r_{\mathrm{N}}^{\mathrm{o}}(t)
$$

According to the theory of receiver function $R(t)=$ $Z(t) \otimes r^{\mathrm{o}}(t)$, we can get

$$
R_{\mathrm{S}}(t)+R_{\mathrm{N}}(t)=\left[Z_{\mathrm{S}}(t)+Z_{\mathrm{N}}(t)\right] \otimes\left[r_{\mathrm{S}}^{\mathrm{o}}(t)+r_{\mathrm{N}}^{\mathrm{o}}(t)\right] .
$$

For the high-SNR records, $Z_{\mathrm{S}}(t) \gg Z_{\mathrm{N}}(t)$ or $Z(t) \approx Z_{\mathrm{S}}(t)$, thus

$$
\begin{gathered}
R_{\mathrm{S}}(t)+R_{\mathrm{N}}(t) \approx Z_{\mathrm{S}}(t) \otimes r_{\mathrm{S}}^{\circ}(t)+Z_{\mathrm{S}}(t) \otimes \\
r_{\mathrm{N}}^{\circ}(t) \approx Z_{\mathrm{S}}(t) \otimes r_{\mathrm{S}}^{\circ}(t)+Z(t) \otimes r_{\mathrm{N}}^{\mathrm{o}}(t) .
\end{gathered}
$$

And for the non-noise ORFs, $R_{\mathrm{S}}(t)=Z_{\mathrm{S}}(t) \otimes r_{\mathrm{S}}^{\mathrm{o}}(t)$, then

$$
R_{\mathrm{N}}(t)=Z(t) \otimes r_{\mathrm{N}}^{\mathrm{o}}(t) .
$$

In this paper we cut the waveforms prior $20 \mathrm{~s}$ to the direct $\mathrm{P}$ as $R_{\mathrm{N}}(t)$, so $r_{\mathrm{N}}^{\mathrm{o}}(t)$ can be obtained by cutting the waveforms on $R_{\mathrm{N}}(t)$ in the time window. The square of the maximum value of $r_{\mathrm{N}}^{\mathrm{o}}(t)$ is considered as the value of $E^{2}$. Then, the velocity error can be calculated by equation (4).

\subsubsection{Error of the depth of discontinuity}

The Gauss filter factor $\alpha$ is equal to 1.5 during the time deconvolution. The resolution of the layer thickness of $r^{\circ}(t)$ with different $\alpha$ values has been discussed by Cassidy (1992), in which the thickness resolution for "410" and "660" were approximately 4.0 and $4.5 \mathrm{~km}$, respectively, for $\alpha=1.5$. These two values are considered as the errors of $h_{410}$ and $h_{660}$ in this study.

\section{Numerical tests of the peeling linear inversion method}

The synthetic receiver functions of the IASP91 model are used to test the method. The steps and parameters are the same as those mentioned in section 2.2. As shown in Figure 1, the depths of $h_{410}$ and $h_{660}$ in the inversion model are 410 and $657 \mathrm{~km}$, respectively, and the mean error of $v_{\mathrm{S}}$ is only $0.052 \mathrm{~km} / \mathrm{s}$, therefore all of the parameters are very close to those of the IASP91 model.

The synthetic receiver function of the preliminary 
reference Earth model (PREM) (Dziewonski and Anderson, 1981) is also used to do the same numerical test as the IASP91. A strong positive signal is present around $24 \mathrm{~s}$, which could be the P-to-S phase for one discontinuity. Thus, $\chi_{i}=5$ is set in the $200-240 \mathrm{~km}$-depth range to determine the discontinuity in the depth range. Figure 2 shows the result of the numerical test. Three discontinuities are found at the depths of 222, 401, and $671 \mathrm{~km}$, respectively, which are close to those of the $\operatorname{PREM}(220,400$, and $670 \mathrm{~km})$. The velocity is also consistent with that of the PREM. The mean error of $v_{\mathrm{S}}$ is $0.079 \mathrm{~km} / \mathrm{s}$.

The results of the above numerical tests indicate that the peeling linear inversion is feasible, and the initial model is general. For example, the inversion result with the initial model based on the IASP91 model is also viable for the synthetic receiver function of PREM.

Similarly, the inversion results of $v_{\mathrm{S}}$ and the depth of the first-order discontinuity are satisfactory in the numerical tests. However, no information is available in the receiver functions for some second-order discontinuities $(\sim 200 \mathrm{~km}$ depth in IASP91 and $\sim 600 \mathrm{~km}$ depth in PREM). Therefore, the method is invalid for secondorder discontinuity due to a large error appearing near the depth in the second-order discontinuity.

\section{Application to the stations GTA, LZH, and AXX}

The linear inversion method mentioned above is applied in three stations, namely, GTA, AXX, and LZH, in northwestern China. We selects the teleseismic waveforms of the events with $M_{\mathrm{S}} \geq 5.5$ and epicentral distance $30^{\circ}-90^{\circ}$ from November 2000 to October 2005 . The corresponding $r^{\circ}(t)$ is then constructed by deconvolving the vertical component from the radial component using an iterative approach (Ligorria and Ammon, 1999). A distance moveout correction for the P-to-S converted phases is adopted (Yuan et al., 1997) to give prominence to $\mathrm{P}_{\mathrm{d}} \mathrm{S}$ (P-to-S phase from the discontinuity to the depth $d$ ) phases for all ORFs. Based on the IASP91 model, all the $\mathrm{P}_{\mathrm{d}} \mathrm{S}$ phases are shifted according to the epicentral distance of $65^{\circ}$ with a $20 \mathrm{~km}$-depth source, then the travel times of $\mathrm{P}_{\mathrm{d}} \mathrm{s}$ phases of different epicentral distance are aligned. Figure 3 shows the stacking results after the moveout correction for the three stations. We can see the clear phases, $\mathrm{P}_{410} \mathrm{~S}$ and $\mathrm{P}_{660} \mathrm{~s}$, from the upper mantle discontinuities in Figure 3.

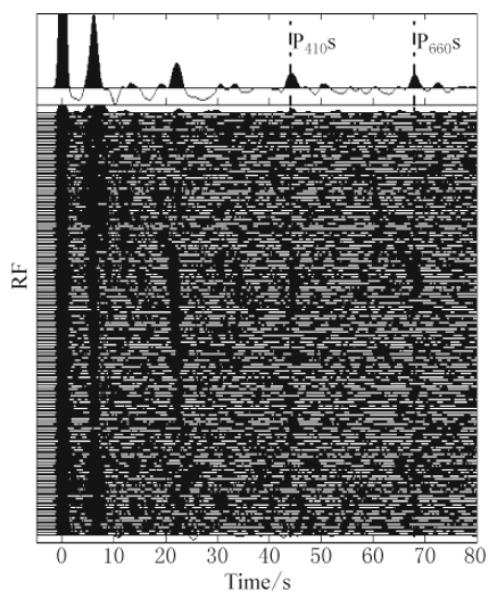

(a)

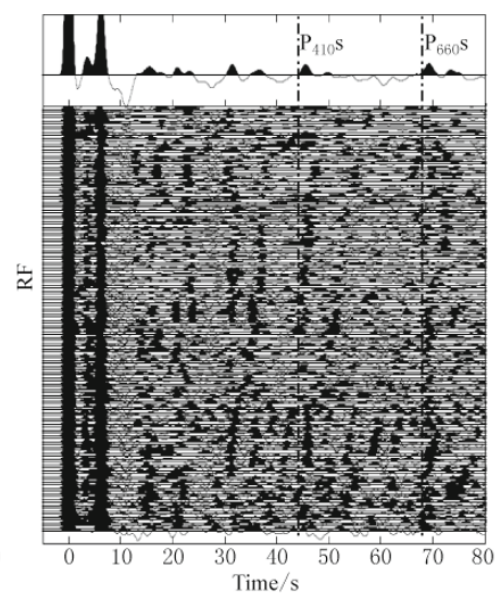

(b)

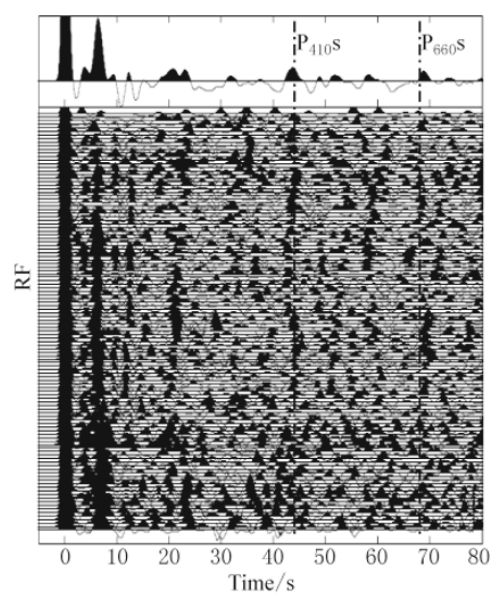

Figure 3 Stacking of the receiver functions after moveout correction of the station GTA with 156 events (a), for the station AXX with 140 events (b), and for the station LZH with 118 events (c).

The GTA station is chosen as an example to describe the data processing and inversion step. The inversion result beneath the station GTA is shown in Figure 4. The initial model is constructed based on the crustal (Ma and Zhou, 2007) and upper mantle models from the surface wave (Huang et al., 2003). Three ORF$\mathrm{s}$ with high SNR are selected to invert the crustal and upper mantle structures with the same step described in section 2.2. Phases $\mathrm{P}_{\mathrm{MS}}, \mathrm{P}_{410} \mathrm{~s}$, and $\mathrm{P}_{660} \mathrm{~s}$ are very clear, thus the smoothness around 51, 410, and $660 \mathrm{~km}$ is very small, whereas the smoothness is large in the other depth ranges. The depths of $h_{410}$ and $h_{660}$ are 398 and $655 \mathrm{~km}$, respectively. The $v_{\mathrm{S}}$ error is $0.023 \mathrm{~km} / \mathrm{s}$ estimated by the method in section 2.3.2.

The same method is used to invert the crustal and upper mantle structures beneath the stations LZH 
(Figure 5) and AXX (Figure 6). Table 1 lists the $h_{410}$, $h_{660}, v_{\mathrm{S}}$ error, and number of $r^{\circ}(t)$ for each station. The possible error from the crust structure will be discussed in the next section (as shown in Figure 7).
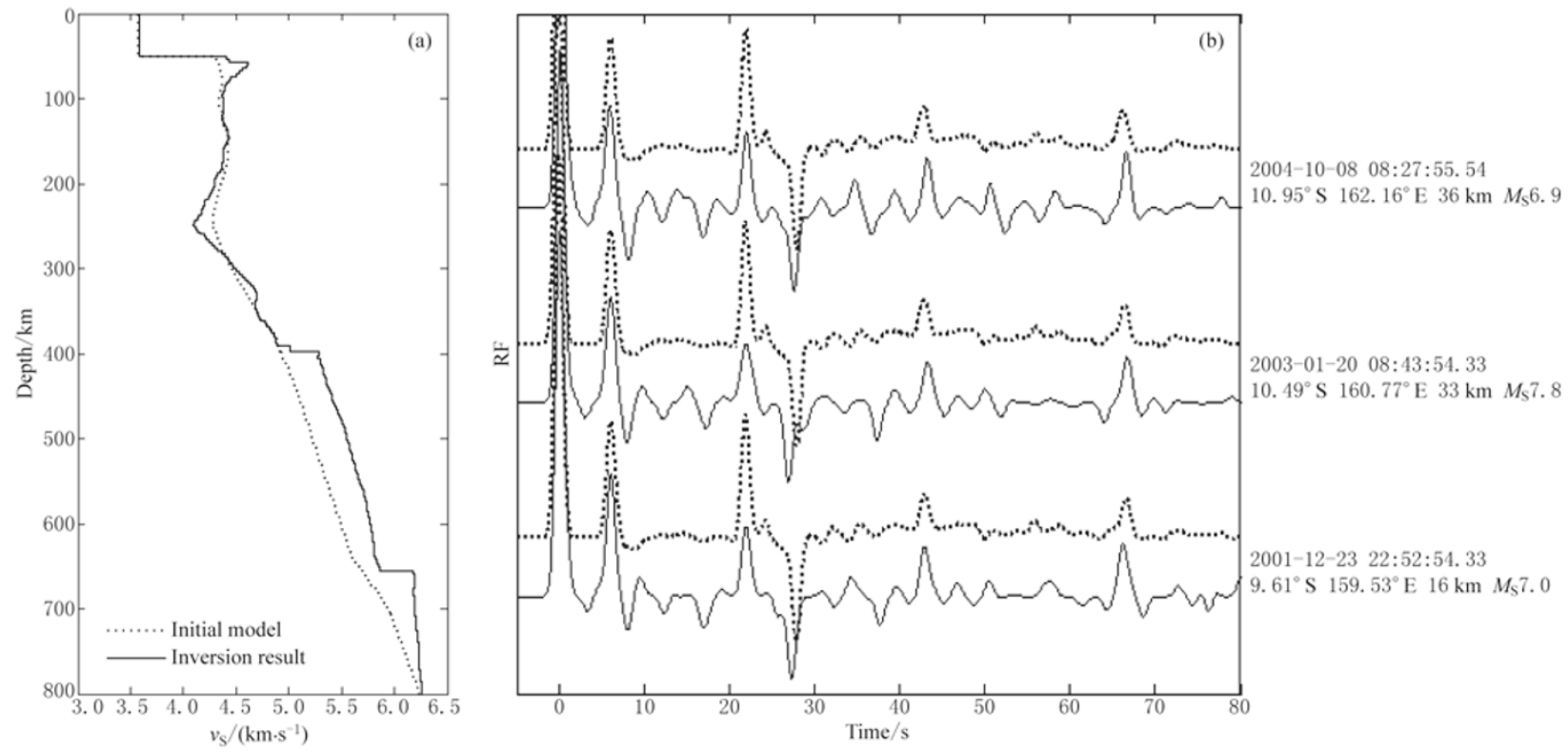

Figure 4 Result of the upper mantle velocity structure with peeling linear inversion method beneath the station GTA. The right panel shows three observed (solid lines) and synthetic (dotted lines) receiver functions. The parameters of the events are also marked. The catalog reference is from U.S. Geological Survey (http://neic.usgs.gov/neis/epic/epic_global.html).
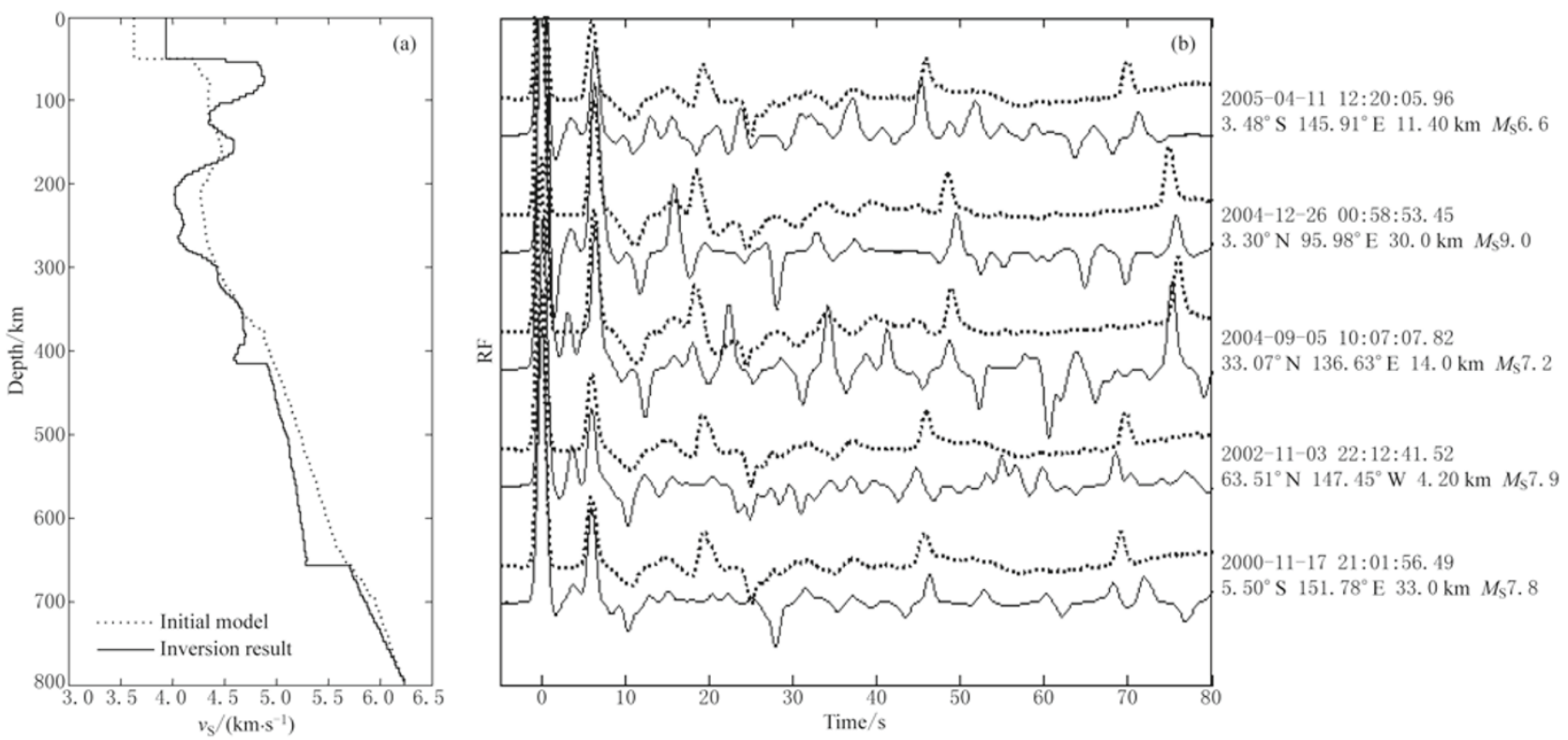

Figure 5 Result of upper mantle velocity structure with peeling linear inversion method beneath the station AXX.

Table 1 The inversion results of $h_{410}, h_{660}, v_{\mathrm{S}}$ error, and number of $r^{\mathrm{o}}(t)$ for three stations

\begin{tabular}{ccccc}
\hline Station & $h_{410} / \mathrm{km}$ & $h_{660} / \mathrm{km}$ & $v_{\mathrm{S}}$ error $/\left(\mathrm{km} \cdot \mathrm{s}^{-1}\right)$ & Number \\
\hline GTA & 398 & 655 & 0.023 & 3 \\
AXX & 416 & 657 & 0.105 & 5 \\
LZH & 415 & 660 & 0.072 & 7 \\
\hline
\end{tabular}



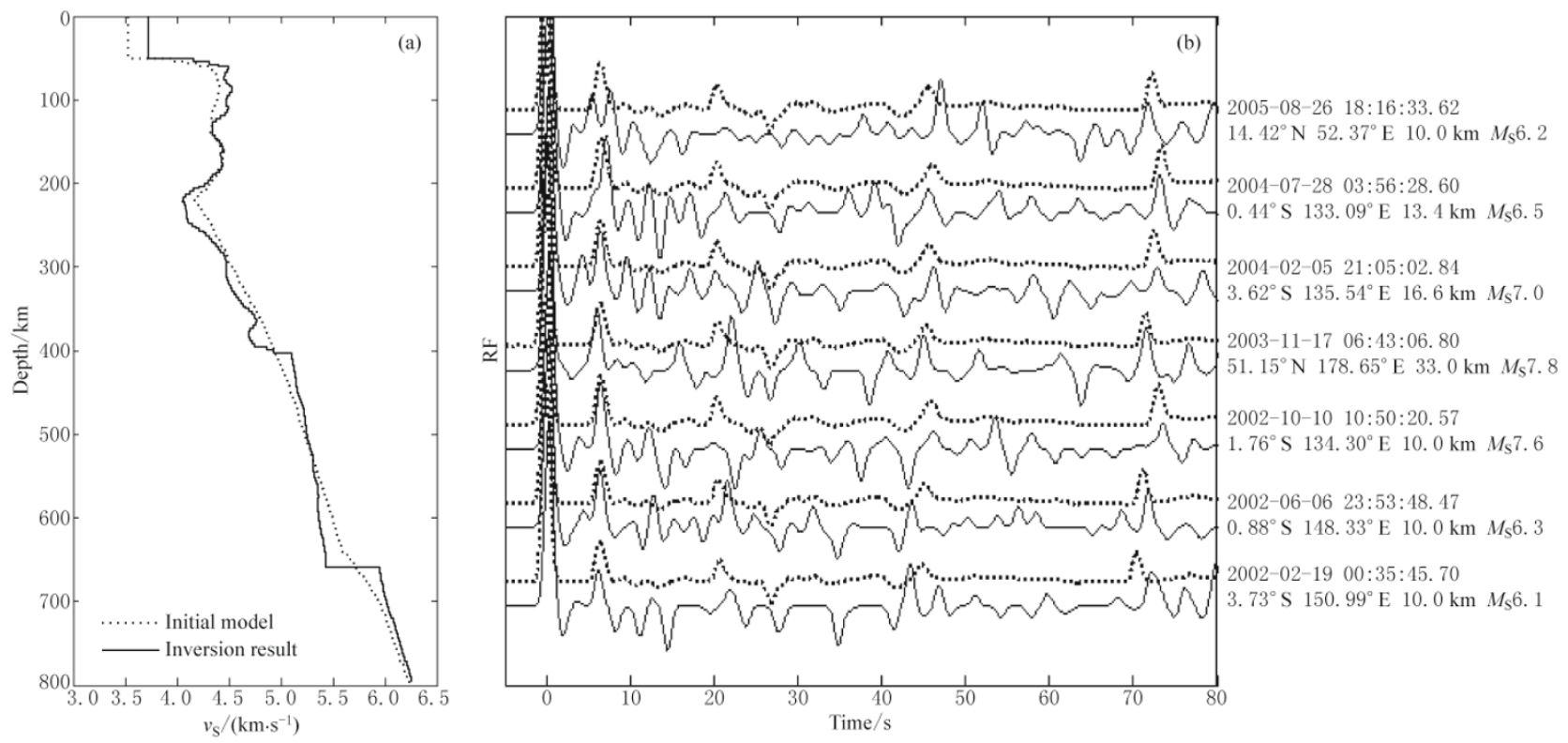

Figure 6 Result of upper mantle velocity structure with peeling linear inversion method beneath the station LZH.
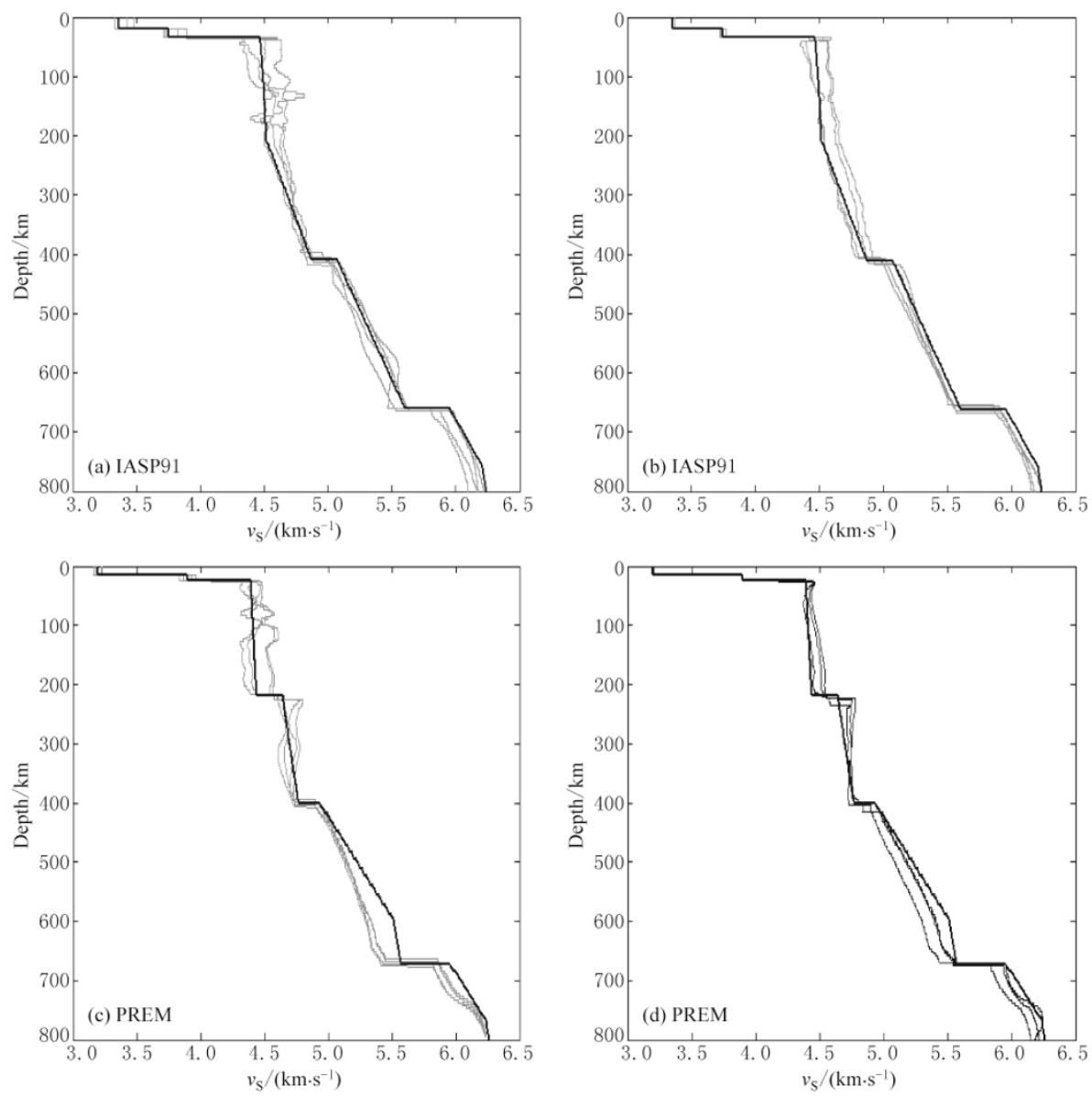

Figure 7 Numerical tests for the error of the crust velocity without (a, c) or with (b, d) smoothing between $3 h_{\mathrm{M}}$ and $5 h_{\mathrm{M}}$. The disturbances with $4 \%$ and $2 \%$ noise are added to the IASP91 and PREM models, respectively. Thick lines denote the true model, and thin lines denote inverted results for different errors. 


\section{Discussion and conclusions}

Some deviations certainly exist between the real crustal structures and the reference model. Such deviation may result from the velocity value, Moho depth, velocity ratio, or all of them. The inversion results are affected by these deviations. The influences from these possible deviations are discussed as follows.

1) Influence from the crustal velocity error. Crustal models with respective errors of $\pm 4 \%$ and $\pm 2 \%$, based on the IASP91 model and PREM, are used to construct the initial model according to the steps outlined in section 2.2.2. The peeling linear method is used to invert the upper mantle structure (Figure $7 \mathrm{a}$ and c). The results indicate that the depth range with the largest error in the inverted results is in the range of $120-190 \mathrm{~km}$ for the IASP91 model and 80-150 km for the PREM. Some false discontinuities exist in this depth range.
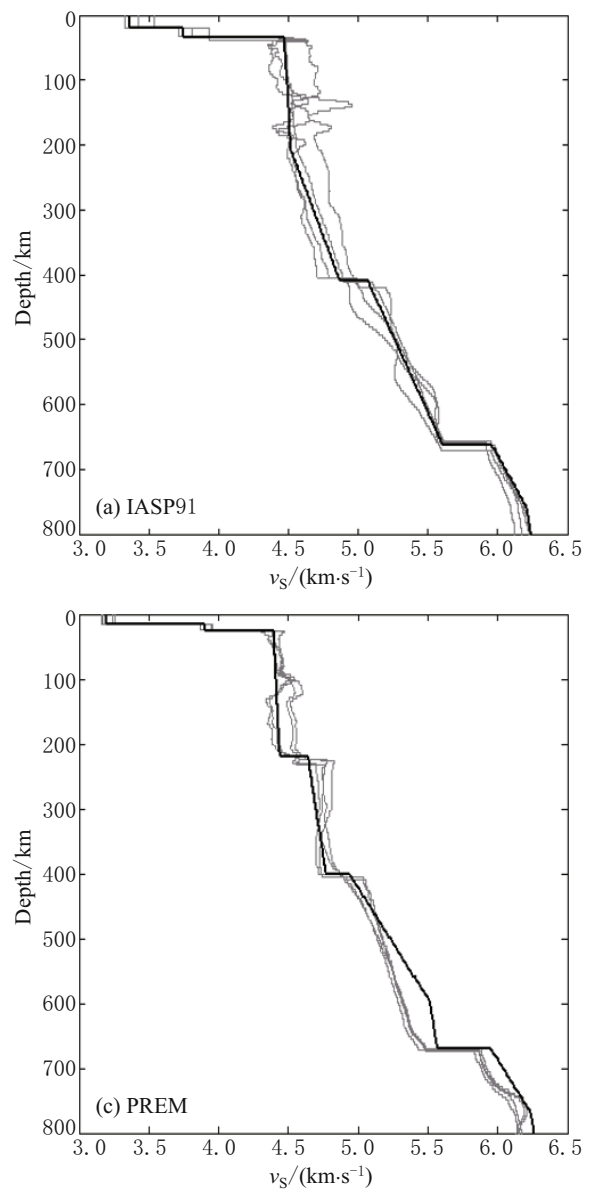

2) Influence from the Moho depth error. The error of the Moho depth $h_{\mathrm{M}}$ is approximately $1-2 \mathrm{~km}$ from the receiver function and the surface wave. Based on the above information, the $h_{\mathrm{M}}$ error model is constructed. $h_{\mathrm{M}}$ changes by values of \pm 2 and $\pm 1 \mathrm{~km}$ based on the IASP91 model and PREM, respectively. The peeling linear method is used to invert the upper mantle structure (Figure 8a and c). The depth ranges with the largest error in the inverted results are still 120-190 km for the IASP91 model and $80-150 \mathrm{~km}$ for the PREM, respectively.

The depth ranges with the largest error are the same for the above two types of error models because the arrival of the multiples in the $r^{\circ}(t)$ from the Moho for the error models deviates from the IASP91 and PREM models. The multiples can be explained by the direct conversion phases from deeper discontinuities; thus, false discontinuities exist in the inverted results
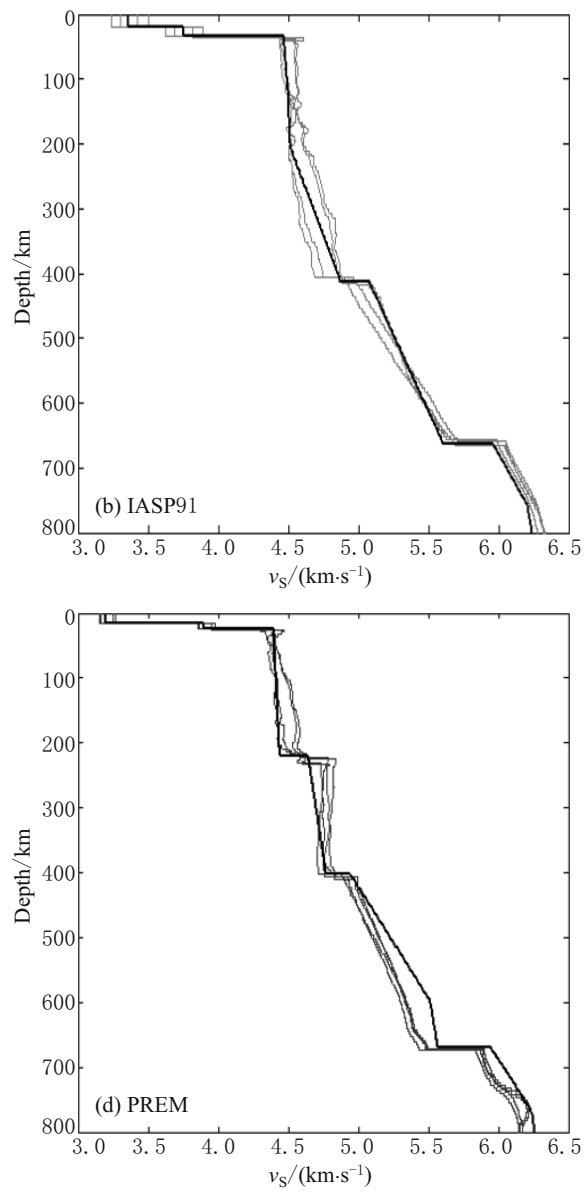

Figure 8 Numerical tests for the error of the Moho depth without (a, c) or with (b, d) smoothing between $3 h_{\mathrm{M}}$ and $5 h_{\mathrm{M}}$. Disturbances with 2 and $1 \mathrm{~km}$ are added to the Moho depth for the IASP91 and PREM models, respectively. Thick lines denote the true model, and thin lines denote inverted results for different errors. 
in the depth range of 120-190 km for the IASP91 model and $80-150 \mathrm{~km}$ for the PREM.

3) Countermeasures to decrease the influences from the crust error. The errors of the crustal model have influence on the inversion results in some depth ranges based on the above numerical tests. The depth range with the largest error is $3 h_{\mathrm{M}}-5 h_{\mathrm{M}}$ in the inversion model. To ensure stability and reliability at this depth range, the previous results are used to construct the initial model. Another valid method is the velocity smoothness $\left(\chi_{i}=0.25\right)$ in the depth range of $3 h_{\mathrm{M}}-5 h_{\mathrm{M}}$. Figures $7 \mathrm{~b}$, $\mathrm{d}$ and $8 \mathrm{~b}, \mathrm{~d}$ show the inversion results with smoothness for the numerical tests mentioned above. The velocity errors become smaller with smoothness. The false discontinuities in the $3 h_{\mathrm{M}}-5 h_{\mathrm{M}}$ depth range also disappear. The detailed velocity structures in this depth range are also smoothed, and the real discontinuities in this depth range cannot be inverted.

4) Influence from the error of $v_{\mathrm{P}} / v_{\mathrm{S}}$ ratio in the upper mantle. The $v_{\mathrm{P}} / v_{\mathrm{S}}$ ratio was fixed in the above method in reference to the IASP91 model. Anyway, the velocity ratio is generally different for IASP91 and the real-Earth models. Zhu and Kanamori (2000) indicated that the estimation of the Moho depth is affected by $v_{\mathrm{P}} / v_{\mathrm{S}}$ ratio. The depths of the upper mantle discontinuities are also influenced by the value of $v_{\mathrm{P}} / v_{\mathrm{S}}$ ratio. Figure 9 shows the ratio in the upper mantle beneath the three stations (GTA, AXX, and LZH) from the $v_{\mathrm{P}}$ and $v_{\mathrm{S}}$ tomography $\left(\mathrm{Qu}\right.$ et al., 2007). The $v_{\mathrm{P}} / v_{\mathrm{S}}$ ratio from the tomography is close to the IASP91 model with a mean error about -0.01 . Therefore, $v_{\mathrm{P}} / v_{\mathrm{S}}$ ratio of the IASP91 model can be used to invert the upper mantle structures for the three stations.

To sum up, in the present paper, the upper mantle structures beneath the three stations (GTA, AXX, and LZH) in northwestern China were inverted using the peeling linear inversion method, which was tested by using the IASP91 and PREM models. Based on the crustal model and prior information from ORFs, the weighted factor $\chi_{i}$ and smoothness matrix $\boldsymbol{\Delta}$ were combined to peel the shallow structure from the Earth model, and the discontinuities were highlighted by suppressing the possible false discontinuities in some depth ranges.

However, the details of the velocity structure in the depth range between $3 h_{\mathrm{M}}$ and $5 h_{\mathrm{M}}$ were lost in the inverted results because of the smoothness. The velocity value was not fixed but varied in a narrow range for $\chi_{i}=0.25$. The initial model refers to the surface tomography in this region, thus the inverted model is reasonable.

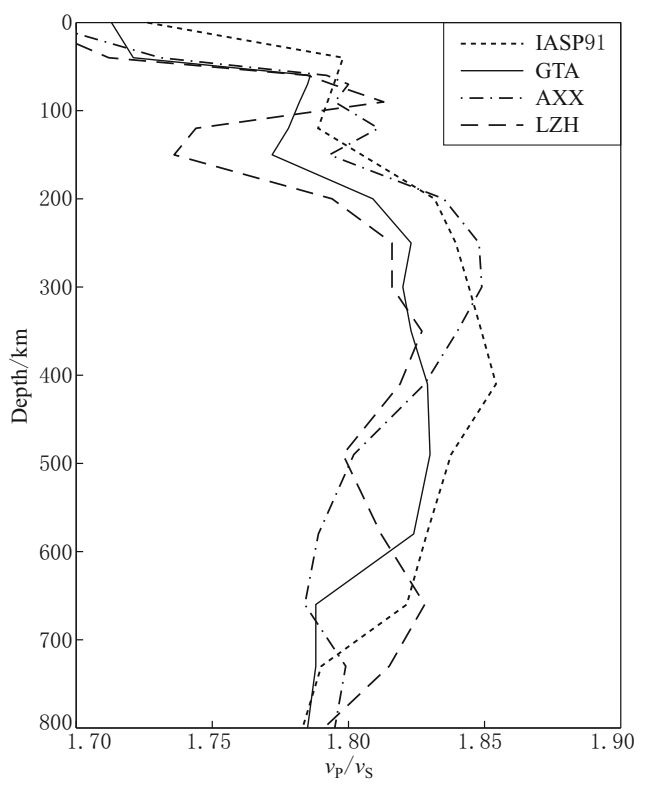

Figure $9 v_{\mathrm{P}} / v_{\mathrm{S}}$ ratio from the tomography with $\mathrm{P}$ and $\mathrm{S}$ phases beneath the three stations. The value of IASP91 is also plotted.

In addition, the inverted result is sensitive to the $v_{\mathrm{P}} / v_{\mathrm{S}}$ ratio. The tomography from the $\mathrm{P}$ and $\mathrm{S}$ phases shows that the difference between the IASP91 and the Earth models beneath the three stations in the present study is very small. The average difference is just -0.012 8. Such difference induces a small error in the result, implying the local model should be used to construct the initial model to reduce the error of the inversion result.

Acknowledgements The authors would like to thank the anonymous reviewers for constructive suggestions. This work was supported by the basic research and development fund from Institute of Earthquake Science, China Earthquake Administration (grant No. 2011IESLZ05) and National Natural Science Foundation of China (grant Nos. 40574024 and 40374009).

\section{References}

Ammon C J, Randall G E and Zandt G (1990). On the nonuniqueness of receiver function inversions. $J$ Geophys Res 95: 15 303-15 318.

Anderson D L and Bass J D (1986). The transition region of the earth upper mantle. Nature 320: 321-328.

Cassidy J F (1992). Numerical experiments in broadband receiver function analysis. Bull Seismol Soc Am 82: 1 4531474.

Dziewonski A M and Anderson D L (1981). Preliminary reference Earth model (PREM). Phys Earth Planet Inter 
25: $297-356$.

Herrmann R B and Ammon C J (2002). Computer programs in seismology: Surface waves, receiver functions and crustal structure. Saint Louis Univ., Saint Louis, MO, USA.

Huang Z, Su W, Peng Y, Zhang Y and Li H (2003). Rayleigh wave tomography of China and adjacent regions. $J$ Geophys Res 108: B22073, doi:10.1029/2001JB001696.

Julià J, Ammon C J and Nyblade A A (2005). Evidence for mafic lower crust in Tanzania, East Africa, from joint inversion of receiver functions and Rayleigh wave dispersion velocities. Geophys J Int 162: 555-569.

Kennett B L N and Engdahl E R (1991). Travel times for global earthquake location and phase identification. Geophys J Int 105: 429-465.

Ligorria J P and Ammon C J (1999). Iterative deconvolution and receiver-function estimation. Bull Seismol Soc Am 89: 1 395-1 400.

Liu Q, Kind R and Li S (1997). The receiver function at the stations of the Chinese digital seismic network (CDSN) and their nonlinear inversion. Chinese J Geophys 40(3): 356-367 (in Chinese with English abstract).

Ma Y and Zhou H (2007). Crustal thicknesses and Poisson's ratios in China by joint analysis of teleseismic receiver functions and Rayleigh wave dispersion. Geophys Res
Lett 34: L12304, doi:10.1029/2007GL029848.

Qu C, Zhou H and Zhao D (2007). Deep structure beneath the west margin of Philippine Sea Plate and South China Sea from $\mathrm{P}$ and $\mathrm{S}$ wave travel time tomography. Chinese $J$ Geophys 50(6): 1 757-1 768 (in Chinese with English abstract).

Shearer P M (1990). Seismic imaging of upper mantle structure and new evidence for a $520 \mathrm{~km}$ discontinuity. Nature 344: 121-126.

Shearer P M and Flanagan M P (1999). Seismic velocity and density jumps across the 410- and 660-kilometer discontinuities. Science 285: 1 545-1 548.

Vinnik L, Reigber C, Aleshin I and Grigoriy L (2004). Receiver function tomography of the central Tien Shan. Earth Planet Sci Lett 225: 131-146.

Yuan X, Ni J, Kind R, Mechie J and Sandvol E (1997). Lithospheric and upper mantle structure of southern Tibet from a seismological passive source experiment. $J$ Geophys Res 102(B12): 27 491-27 500.

Zhou H and Yang Y (2003). Peeling genetic algorithm of receiver functions inversion for upper mantle structure and discontinuities. Chinese J Geophys 46(3): 546-556.

Zhu L and Kanamori H (2000). Moho depth variation in southern California from teleseismic receiver functions. J Geophys Res 105: 2 969-2 980. 